\title{
A direct numerical integration scheme for visco-hyperelastic models using radial return relaxation
}

\author{
Stéphane Lejeunes* — Stéphane Méo** — Adnane Boukamel*,**** \\ * Laboratoire de Mécanique et d'Acoustique de Marseille (CNRS UPR7051) \\ 31 ch Joseph-Aiguier, F-13402 Marseille \\ lejeunes@lma.cnrs-mrs.fr \\ ** Laboratoire de Mécanique et Rhéologie de l'Université de Tours \\ 7 Avenue Marcel Dassault, F-37200 Tours \\ stephane.meo@univ-tours.fr \\ *** Ecole Centrale Marseille \\ Technopôle Château Gombert, F-13451 Marseille \\ adnane.boukamel@ec-marseille.fr
}

\begin{abstract}
In this paper, a numerical integration scheme of the evolution laws for viscohyperelastic models is proposed. The starting points of the method are the exponential mapping (Reese et al., 1998) and the radial return (Weber et al., 1990; Simo, 1988). The originality of this work lies in the substitution of a differential tensorial system by a scalar one with two equations and two unknowns and in a first order Taylor expansion of them. In this way an analytical approximated exponential solution is finally obtained.

RÉSUMÉ. On propose dans cet article un schéma numérique d'intégration de lois d'évolutions visco-hyperélastiques s'inspirant à la fois des méthodes par retour en exponentiel (Reese et al., 1998) et des méthodes par retour radial (Weber et al., 1990 ; Simo, 1988). L'originalité de ce schéma repose à la fois sur la substitution d'un système différentiel tensoriel, traduisant les lois d'évolutions, par un système différentiel scalaire de deux équations à deux inconnues, et sur un développement limité au premier ordre de ces dernières. On obtient ainsi une solution analytique approchée en exponentiel.

KEYWORDS: radial return relaxation, visco-hyperelasticity, finite element method. MOTS-CLÉS : relaxation radiale, visco-hyperélasticité, méthode des éléments finis.
\end{abstract}

DOI:10.3166/EJCM.19.129-140 (C) 2010 Lavoisier, Paris

EJCM - 19/2010. Giens 2009, pages 129 to 140 


\section{Introduction}

In the past twenty years, the modeling of polymer materials has received a very large consideration in the scientific community. Many finite strain rheological models, which are based on the intermediate states principle, have been proposed. They are often seen as a generalization of small strain models and for most of them, a numerical implementation has already been proposed. Nevertheless many of the existing schemes exhibit limitations that are not always discussed. As the complexity of these models has growing in the last years, the question of an efficient and generic numerical scheme is still open.

The originality of the present paper lies in an approximation of the evolution laws by a scalar and linear differential system. This approximation is obtained by using a decomposition of the internal tensorial variables into direction and magnitude. In the case of associative plasticity with $J 2$-flow the problem is reduced to the determination of a scalar parameter. This process is known as the radial-return algorithm, (Simo, 1988; Simo et al., 2000). In the case of viscoelasticity the internal variables evolution is generally fully tensorial and thus can not be reduced to a scalar equation. Besides the class of tensorial integrators, some authors developed specific algorithms based on exponential mapping (Weber et al., 1990). In (Reese et al., 1998) this method is developed on a Zener model. By considering the evolution law in the principal space and using the logarithmic principal stretches, the integration is reduced to the determination of a non-linear system of three equations which is easily solved by a local newton scheme. This scheme is very robust and since the earlier paper of (Reese et al., 1998), many authors have used this strategy (see for examples (Nedjar, 2002a; Nedjar, 2002b; Fancello et al., 2008; Areias et al., 2008). In this paper, it is proposed to keep the flow direction fixed during a step of integration and to linearize the differential system around the previous solution obtained. The system obtained is then easily integrated with an analytical solution which takes the form of an infinite Magnus series.

This paper is organized as follows, the constitutive equations of a Zener model are briefly recalled in a first section. The variational formulation and the finite element implementation is described in the second section. In a third section, the numerical integration scheme is detailed. Finally, the numerical performance of the proposed scheme is discussed in the last section.

\section{Constitutive equations}

Assuming that the Clausius-Duhem inequality can be written in the eulerian configuration (if the thermal phenomena are neglected):

$$
\phi=\boldsymbol{\sigma}: \mathbf{D}-J^{-1} \rho_{0} \dot{\psi} \geq 0
$$

where $\boldsymbol{\sigma}, \mathbf{D}, J, \rho_{0}$ and $\psi$ are respectively the Cauchy stress tensor, the eulerian rate of deformation, the determinant of $\mathbf{F}$ (see Figure 1) the gradient of the considered 


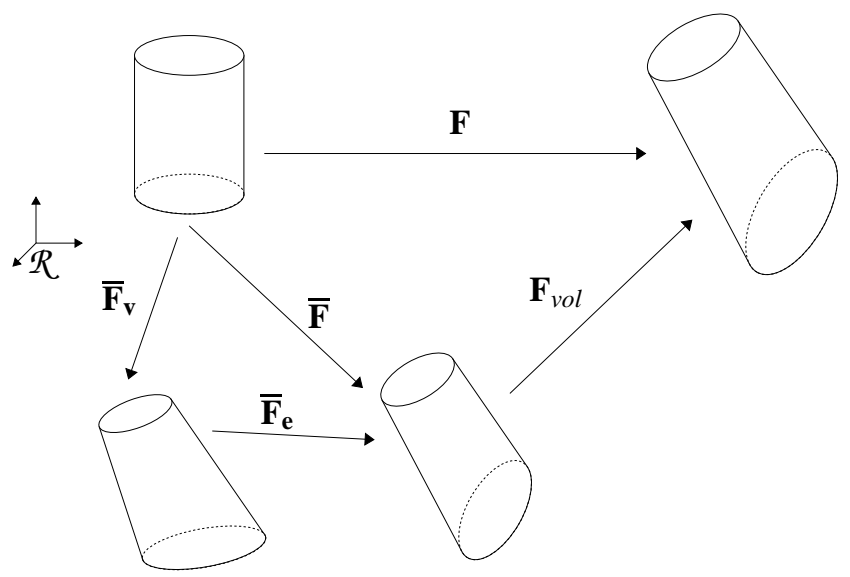

Figure 1. Intermediate states and decomposition of the gradient tensor

transformation, the volumic mass in the lagrangian configuration and the free specific energy.

Then the gradient of the transformation is split into an incompressible part $(\overline{\mathbf{F}}=$ $\left.J^{-\frac{1}{3}} \mathbf{F}\right)$ and into a compressible one $\left(\mathbf{F}_{\text {vol }}\right)$ leading to see $\psi$ as a function of $\overline{\mathbf{F}}$ and $J$. The free specific energy is then additively decomposed into an isochoric part and a volumetric one:

$$
\psi(\overline{\mathbf{F}}, J)=\bar{\psi}(\overline{\mathbf{F}})+\psi_{\text {vol }}(J)
$$

Now, using the concept of intermediate states (Sidoroff, 1973; Sidoroff, 1975) introducing a viscous intermediate state (see Figure 1), $\overline{\mathbf{F}}$ the incompressible gradient is also split into a viscous part and an elastic part:

$$
\overline{\mathbf{F}}=\overline{\mathbf{F}}_{\mathbf{e}} \cdot \overline{\mathbf{F}}_{\mathbf{v}}
$$

Some considerations of objectivity and isotropy lead to have $\bar{\psi}$ as a function of $\overline{\mathbf{B}}$ and $\overline{\mathbf{B}}_{\mathbf{e}}$, the left Cauchy Green tensors ${ }^{1}$ associated to respectively $\overline{\mathbf{F}}$ and $\overline{\mathbf{F}}_{\mathbf{e}}$ (Sidoroff, 1975; Sidoroff, 1976). It is then chosen to see $\bar{\psi}$ as the sum :

$$
\bar{\psi}\left(\overline{\mathbf{B}}, \overline{\mathbf{B}}_{\mathbf{e}}\right)=\psi_{v}\left(\overline{\mathbf{B}}_{\mathbf{e}}\right)+\psi_{h}(\overline{\mathbf{B}})
$$

Considering $\left(\mathbf{B}, \overline{\mathbf{B}}_{\mathbf{e}}, J\right)$ as an independent set of parameters, [4] and [2] give the variation of $\psi^{2}$

$$
\dot{\psi}=\frac{\partial \psi_{h}}{\partial \overline{\mathbf{B}}}: \frac{\partial \overline{\mathbf{B}}}{\partial \mathbf{B}}: \dot{\mathbf{B}}+\frac{\partial \psi_{v}}{\partial \overline{\mathbf{B}}_{\mathbf{e}}}: \dot{\overline{\mathbf{B}}}_{\mathbf{e}}+\frac{\partial \psi_{v o l}}{\partial J} \dot{J}
$$

1. $\overline{\mathbf{B}}=\overline{\mathbf{F}} \cdot \overline{\mathbf{F}}^{T} \quad \overline{\mathbf{B}}_{\mathbf{e}}=\overline{\mathbf{F}}_{\mathbf{e}} \cdot \overline{\mathbf{F}}_{\mathbf{e}}^{T}$

2. the operators : and $\cdot$ are defined in appendix. 
The time derivatives of $\mathbf{B}, \overline{\mathbf{B}}_{\mathbf{e}}$ and $J$ are given by:

$$
\begin{aligned}
& \dot{\mathbf{B}}=\mathbf{L} \cdot \mathbf{B}+\mathbf{B} \cdot \mathbf{L}^{T} \\
& \dot{J}=J(\mathbf{1}: L) \\
& \dot{\overline{\mathbf{B}}}_{\mathbf{e}}=\mathbf{L} \cdot \overline{\mathbf{B}}_{\mathbf{e}}+\overline{\mathbf{B}}_{\mathbf{e}} \cdot \mathbf{L}^{T}-2 \overline{\mathbf{V}}_{\mathbf{e}} \cdot \overline{\mathbf{D}}_{\mathbf{v}}^{\mathbf{o}} \cdot \overline{\mathbf{V}}_{\mathbf{e}}-\frac{2}{3}(\mathbf{1}: \mathbf{D}) \overline{\mathbf{B}}_{\mathbf{e}}
\end{aligned}
$$

with $\mathbf{L}=\dot{\mathbf{F}} \cdot \mathbf{F}^{-1}$ and $\overline{\mathbf{V}}_{\mathbf{e}}$ being the pure eulerian strain tensor ${ }^{3}$. [8] also introduced an objective anelastic rate of deformation $\overline{\mathbf{D}}_{\mathbf{v}}^{\mathbf{o}}$

$$
\overline{\mathbf{D}}_{\mathbf{v}}^{\mathbf{o}}=\overline{\mathbf{R}}_{\mathbf{e}} \cdot\left(\dot{\mathbf{F}}_{\mathbf{v}} \cdot \overline{\mathbf{F}}_{\mathbf{v}}{ }^{-1}\right)_{s y m} \cdot \overline{\mathbf{R}}_{\mathbf{e}}^{T}=\overline{\mathbf{R}}_{\mathbf{e}} \cdot \overline{\mathbf{D}}_{\mathbf{v}} \cdot \overline{\mathbf{R}}_{\mathbf{e}}{ }^{T}
$$

Here, it is supposed that all the rotation obtained from the decomposition of $\overline{\mathbf{F}}$ is due to the elastic transformation i.e.

$$
\overline{\mathbf{R}}=\overline{\mathbf{R}}_{\mathbf{e}} \text { and so } \overline{\mathbf{R}}_{\mathbf{v}}=\mathbf{1} \text {. }
$$

At this step, we can write using [6], [7] and [8] in [5]:

$$
\begin{aligned}
\dot{\psi}=\left(\left(\frac{\partial \psi_{h}}{\partial \overline{\mathbf{B}}}: \frac{\partial \overline{\mathbf{B}}}{\partial \mathbf{B}}\right) \cdot \mathbf{B}\right): \mathbf{D} & +\left(2 \overline{\mathbf{B}}_{\mathbf{e}} \frac{\partial \psi_{v}}{\partial \overline{\mathbf{B}}_{\mathbf{e}}}\right)^{D}: \mathbf{D}+J\left(\frac{\partial \psi_{v o l}}{\partial J} \mathbf{1}\right): \mathbf{D} \\
& -\left(2 \overline{\mathbf{V}}_{\mathbf{e}} \cdot \frac{\partial \psi_{v}}{\partial \overline{\mathbf{B}}_{\mathbf{e}}} \cdot \overline{\mathbf{V}}_{\mathbf{e}}\right): \overline{\mathbf{D}}_{\mathbf{v}}^{\mathbf{o}}
\end{aligned}
$$

Observing the relationship

$$
\left(\left(\frac{\partial \psi_{h}}{\partial \overline{\mathbf{B}}}: \frac{\partial \overline{\mathbf{B}}}{\partial \mathbf{B}}\right) \cdot \mathbf{B}\right): \mathbf{D}=\left(\overline{\mathbf{B}} \cdot \frac{\partial \psi_{h}}{\partial \overline{\mathbf{B}}}\right)^{D}: \mathbf{D}
$$

and under the assumption of a normal dissipation only depending on $\overline{\mathbf{D}}_{\mathbf{v}}^{\mathbf{0}}$, the behavior and the complementary laws are obtained:

$$
\left\{\begin{array}{l}
\boldsymbol{\sigma}=\boldsymbol{\sigma}_{\boldsymbol{h}}+\boldsymbol{\sigma}_{\boldsymbol{v}}+\boldsymbol{\sigma}_{v o l} \\
2 \rho_{0} J^{-1}\left(\overline{\mathbf{V}}_{\mathbf{e}} \cdot \frac{\partial \psi_{v}}{\partial \overline{\mathbf{B}}_{\mathbf{e}}} \cdot \overline{\mathbf{V}}_{\mathbf{e}}\right)^{D}=\frac{\partial \varphi_{v}}{\partial \overline{\mathbf{D}}_{\mathbf{v}}^{\mathbf{0}}} \\
\boldsymbol{\sigma}_{\boldsymbol{h}}=2 \rho_{0} J^{-1}\left(\overline{\mathbf{B}} \cdot \frac{\partial \psi_{h}}{\partial \overline{\mathbf{B}}}\right)^{D} \\
\boldsymbol{\sigma}_{v o l}=p \mathbf{1} \text { with } p=\rho_{0} \frac{\partial \psi_{v o l}}{\partial J} \\
\boldsymbol{\sigma}_{\boldsymbol{v}}=2 \rho_{0} J^{-1}\left(\overline{\mathbf{B}}_{\mathbf{e}} \cdot \frac{\partial \psi_{v}}{\partial \overline{\mathbf{B}}_{\mathbf{e}}}\right)^{D}
\end{array}\right.
$$

Equations [13] can be seen as a generalization to large strain of the classical Zener rheological model (Figure 2) in the incompressible case.

3. $\overline{\mathbf{V}}_{\mathbf{e}}$ is defined by the polar decomposition $\overline{\mathbf{F}}_{\mathbf{e}}=\overline{\mathbf{V}}_{\mathbf{e}} \cdot \overline{\mathbf{R}}_{\mathbf{e}}$ and in the same way $\overline{\mathbf{F}}_{\mathbf{v}}=\overline{\mathbf{V}}_{\mathbf{v}} \cdot \overline{\mathbf{R}}_{\mathbf{v}}$ 


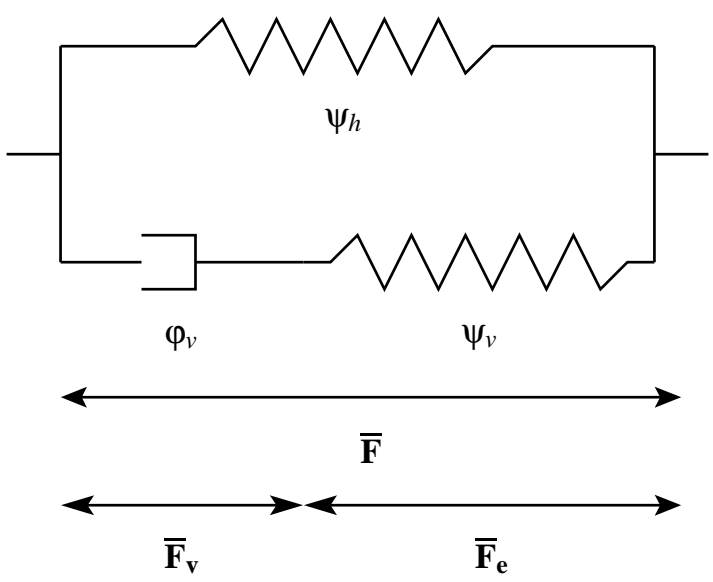

Figure 2. Zener model

\section{Variational formulation and finite element implementation}

The quasi-incompressibility constraint can be applied using a perturbed lagrangian method. The solution $(\mathbf{u} ; p)$ of the equilibrium problem has to cancel the following integral forms for all the trial functions $\delta \mathbf{v}$ and $\delta p$ chosen respectively in the same spaces of $\mathbf{u}$ and $p$.

$$
\left\{\begin{array}{l}
\int_{\Omega_{0}} \pi: \nabla \delta v d \Omega-\int_{\Omega_{0}} \delta v \cdot \mathbf{f}_{e x t} d \Omega-\int_{\delta \Omega} \delta v \cdot \mathbf{F}_{e x t} d S \\
\int_{\Omega_{0}}\left(-(J(\mathbf{u})-1)-\frac{1}{k} p\right) \delta q d \Omega
\end{array}\right.
$$

where $\boldsymbol{\pi}$ is the first Piola-Kirchoff stress tensor, $\mathbf{f}_{\text {ext }}$ and $\mathbf{F}_{\text {ext }}$ are respectively the volumic external forces and the surfacic ones. The second equation results from the implicit choice of $\rho_{0} \psi_{v o l}$ as:

$$
\rho_{0} \psi_{v o l}=\frac{k}{2}(J-1)^{2}
$$

System [14] is handled using a Newton-Raphson algorithm leading to the calculation of the tangent moduli:

$$
\mathbb{T}=\frac{\partial^{2} \psi}{\partial \mathbf{F}^{2}}=\frac{\partial \boldsymbol{\pi}}{\partial \mathbf{F}}=\mathbb{T}_{\mathbf{h}}+\mathbb{T}_{\mathbf{v}}+\mathbb{T}_{\mathbf{p}}
$$

and

$$
\left\{\begin{array}{l}
\mathbb{T}_{\mathbf{h}}=\frac{\partial \boldsymbol{\pi}_{\text {hyp }}}{\partial \mathbf{F}} \text { where } \boldsymbol{\pi}_{\text {hyp }}=J\left(\boldsymbol{\sigma}_{h}+\boldsymbol{\sigma}_{\text {vol }}\right) \cdot \mathbf{F}^{-\mathbf{T}} \\
\mathbb{T}_{\mathbf{v}}=\frac{\partial \boldsymbol{\pi}_{\boldsymbol{v}}}{\partial \mathbf{F}} \text { where } \boldsymbol{\pi}_{v}=J \boldsymbol{\sigma}_{v} \cdot \mathbf{F}^{-\mathbf{T}}
\end{array}\right.
$$


For reason of simplicity, all the implementations are made in an oriented object F.E. code (here a $C^{++}$code) (Foerch et al., 1996). A visco-elastic behavior class is created. It inherits from an existing hyperelastic $C^{++}$behavior class in which the calculations of $\boldsymbol{\pi}_{\text {hyp }}$ and $\mathbb{T}_{\mathbf{h}}$ are already carried out (Lejeunes, 2006).

\section{Visco-hyperelastic behaviour integration}

\subsection{Flow rule}

At this step, the potentials entirely determine the behavior law [13], they are chosen such that $\left(\rho_{0} \psi_{\text {vol }}\right.$ being already chosen in [15]):

$$
\left\{\begin{array}{l}
\rho_{0} \psi_{h}(\overline{\mathbf{B}})=C_{10}\left(I_{1}(\overline{\mathbf{B}})-3\right)+C_{01} \ln \left(\frac{I_{2}(\overline{\mathbf{B}})}{3}\right) \\
\rho_{0} \psi_{v}\left(\overline{\mathbf{B}}_{\mathbf{e}}\right)=G\left(I_{1}\left(\overline{\mathbf{B}}_{\mathbf{e}}\right)-3\right) \\
\varphi_{v}\left(\overline{\mathbf{D}}_{\mathbf{v}}^{\mathbf{o}}\right)=\frac{\eta}{2}\left(\overline{\mathbf{D}}_{\mathbf{v}}^{\mathbf{o}}: \overline{\mathbf{D}}_{\mathbf{v}}^{\mathbf{o}}\right)
\end{array}\right.
$$

where $C_{10}, C_{01}, G$ and $\eta$ are 4 material parameters.

Equation [18] leads to, using [13] and [8] with $\tau=\frac{4 G}{J \eta}$ :

$$
\dot{\overline{\mathbf{B}}}_{\mathbf{e}}=\mathbf{L} \cdot \overline{\mathbf{B}}_{\mathbf{e}}+\overline{\mathbf{B}}_{\mathbf{e}} \cdot \mathbf{L}-\tau \overline{\mathbf{V}}_{\mathbf{e}} \cdot \overline{\mathbf{B}}_{\mathbf{e}}{ }^{D} \cdot \overline{\mathbf{V}}_{\mathbf{e}}-\frac{2}{3}(\mathbf{1}: \mathbf{L}) \overline{\mathbf{B}}_{\mathbf{e}}
$$

Having in mind that $\overline{\mathbf{V}}_{\mathbf{e}} \cdot \overline{\mathbf{B}}_{\mathbf{e}}{ }^{D} \cdot \overline{\mathbf{V}}_{\mathbf{e}}=\overline{\mathbf{B}}_{\mathbf{e}}{ }^{D} \cdot \overline{\mathbf{B}}_{\mathbf{e}}$, [19] comes to be known as:

$$
\dot{\overline{\mathbf{B}}}_{\mathbf{e}}=\mathbf{L} \cdot \overline{\mathbf{B}}_{\mathbf{e}}+\overline{\mathbf{B}}_{\mathbf{e}} \cdot \mathbf{L}-\tau \overline{\mathbf{B}}_{\mathbf{e}}{ }^{D} \cdot \overline{\mathbf{B}}_{\mathbf{e}}-\frac{2}{3}(\mathbf{1}: \mathbf{L}) \overline{\mathbf{B}}_{\mathbf{e}}
$$

\subsection{Numerical scheme and elastic prediction}

In this section, the evolution of [20] will be considered on the time interval $\left[t_{n}, t_{n+1}\right], t_{n}$ being the last converged time increment of the Newton-Raphson algorithm. So, all the variables are supposed to be known at this time. Moreover $\mathbf{F}$ is supposed to have a linear temporal evolution (i.e. $\dot{\mathbf{F}}$ is constant).

At $t \in\left[t_{n}, t_{n+1}\right]$, let's define an elastic trial state as ${ }^{4}$ :

$$
\overline{\mathbf{F}}_{\mathbf{e}}^{\star}=\overline{\mathbf{F}} \cdot \overline{\mathbf{F}}_{\left.\mathbf{v}\right|_{n}}^{-1}
$$

and thus the prediction of the left Cauchy-Green related to $\overline{\mathbf{F}}_{\mathbf{e}}{ }^{\star}$ is:

$$
\overline{\mathbf{B}}_{\mathbf{e}}^{\star}=\overline{\mathbf{F}} \cdot \overline{\mathbf{F}}_{\left.\right|_{n}} \cdot \overline{\mathbf{B}}_{\left.\mathbf{e}\right|_{n}} \cdot \overline{\mathbf{F}}_{\left.\right|_{n}}^{-T} \cdot \overline{\mathbf{F}}^{T}
$$

4. The subscript ${ }_{-\left.\right|_{n}}$ means the value of the variable at the time $t_{n},{ }_{-\left.\right|_{n+1}}$ at $t_{n+1}$ and without subscript it is considered at $t$. 
The trial deviatoric stress tensor is then:

$$
\boldsymbol{\sigma}_{\boldsymbol{v}}{ }^{\star}=2 J^{-1} G \overline{\mathbf{B}}_{\mathbf{e}} \star^{D}
$$

This allows to define the normalised deviatoric stress tensor ${ }^{5}$ :

$$
\mathbf{N}^{\star}=\frac{\boldsymbol{\sigma}_{v}{ }^{\star}}{\left\|\boldsymbol{\sigma}_{\boldsymbol{v}}{ }^{\star}\right\|}=\frac{\overline{\mathbf{B}}_{\mathbf{e}}{ }^{{ }^{D}}}{\left\|\overline{\mathbf{B}}_{\mathbf{e}}{ }^{{ }^{D}}\right\|}
$$

By definition we have:

$$
\mathbf{N}^{\star}: \mathbf{N}^{\star}=1, \mathbf{N}^{\star}: \mathbf{1}=0
$$

This elastic trial state corresponds exactly to the solution with $\tau=0$. It is the starting point of the mapping exponential methods (Reese et al., 1998; Nedjar, 2002b; Nedjar, 2002a), where it is relaxed to satisfy the flow rule. During this relaxation the viscous variable is frozen in order to obtain an exponential solution. In our study, we rather draw our inspiration from the radial return mapping developed in plasticity (Simo, 1988) leading to suppose that:

$$
\overline{\mathbf{B}}_{\mathbf{e}}=b(t) \mathbf{N}^{\star}+s(t) \mathbf{1}
$$

with the functions $b(t)$ and $s(t)$ defined as:

$$
b(t)=\overline{\mathbf{B}}_{\mathbf{e}}: \mathbf{N}^{\star} \quad s(t)=\frac{1}{3} \overline{\mathbf{B}}_{\mathbf{e}}: \mathbf{1}
$$

$\dot{\overline{\mathbf{B}}}_{\mathbf{e}}$ is expressible as:

$$
\dot{\overline{\mathbf{B}}}_{\mathbf{e}}=\dot{b} \mathbf{N}^{\star}+b \dot{\mathbf{N}}^{\star}+\dot{s} \mathbf{1}
$$

Taking into account [26] and [28], the projections of [20] (using $\mathbf{1}$ and $\mathbf{N}^{\star}$ as projectors) give

$$
\left\{\begin{aligned}
\dot{s}= & \frac{2}{3}\left(\mathbf{L}: \mathbf{N}^{\star}\right) b-\frac{\tau}{3} b^{2} \\
\dot{b}= & \left(2\left(\mathbf{L}: \mathbf{N}^{\star^{2}}\right)-\frac{2}{3}(\mathbf{1}: \mathbf{L})\right) b+2\left(\mathbf{L}: \mathbf{N}^{\star}\right) s \\
& -\tau\left(\left(\mathbf{N}^{\star^{2}}: \mathbf{N}^{\star}\right) b^{2}+s b\right)
\end{aligned}\right.
$$

The system [29] is a scalar non linear differential system (with non-constant coefficients). It can be solved using a Runge-Kutta method or any other $\theta$-scheme for example. It is not our choice, but in order to compare with the chosen procedure, a Runge-Kutta-Fehlberg allowing to control the step size of the numerical iterative scheme is implemented (Fehlberg, 1969).

5. $\left\|_{-}\right\|$is defined in appendix. 


\subsection{First order expansion and exponential mapping}

The proposed approach consists in a first order Taylor expansion of the system [29] around the last converged solution $\left(b_{n}=b\left(t_{n}\right), s_{n}=s\left(t_{n}\right)\right)$ :

$$
b(t)=b_{n}+\delta b \quad s(t)=s_{n}+\delta s
$$

Carrying forward these approximations in [29], it is obtained:

$$
\left\{\begin{array}{c}
\dot{\delta} s \\
\dot{\delta} b
\end{array}\right\}=[A(t)]\left\{\begin{array}{l}
\delta s \\
\delta b
\end{array}\right\}+\{C(t)\}
$$

with the initial condition:

$$
\left\{\begin{array}{l}
\delta s\left(t_{n}\right) \\
\delta b\left(t_{n}\right)
\end{array}\right\}=\left\{\begin{array}{l}
0 \\
0
\end{array}\right\}
$$

where $[A(t)]$ and $\{C(t)\}$ are respectively a $2 \times 2$ matrix and a two components vector defined by:

$$
\begin{aligned}
& A_{11}=0 \quad A_{12}=\frac{2}{3}\left(\mathbf{L}: \mathbf{N}^{\star}\right)-\frac{2 \tau}{3} b_{n} \\
& A_{21}=2\left(\mathbf{L}: \mathbf{N}^{\star}\right)-\tau b_{n} \quad A_{22}=2\left(\mathbf{L}: \mathbf{N}^{\star^{2}}\right)-2 \tau b_{n}\left(\mathbf{N}^{\star^{2}}: \mathbf{N}^{\star}\right) \\
& -\tau s_{n}-\frac{2}{3}(\mathbf{1}: \mathbf{L}) \\
& C_{1}=\left(2\left(\mathbf{L}: \mathbf{N}^{\star}\right)-\tau b_{n}\right) \frac{b_{n}}{3} \quad C_{2}=2\left(L: \mathbf{N}^{\star^{2}}\right) b_{n}+\left(\mathbf{L}: \mathbf{N}^{\star}\right) s_{n} \\
& -\frac{2}{3}(1: \mathbf{L}) b_{n}-\tau b_{n}^{2}
\end{aligned}
$$

The solution of this differential system is:

$$
\left\{\begin{array}{l}
\delta s\left(t_{n+1}\right) \\
\delta b\left(t_{n+1}\right)
\end{array}\right\}=\int_{t_{n}}^{t_{n+1}} e^{\left[\Omega\left(t_{n+1}-s\right)\right]}\{C(s)\} d s
$$

where $\Omega(t)$ is a Magnus series expansion (Iserles. et al., 1999):

$$
\begin{aligned}
\Omega(t) & =\int_{t_{n}}^{t} A(s) d s+\frac{1}{2} \int_{t_{n}}^{t}\left[A(s), \int_{t_{n}}^{t} A(s 1) d s 1\right] d s \\
& +\frac{1}{4} \int_{t_{n}}^{t}\left[A(s), \int_{t_{n}}^{t}\left[A(s 1), \int_{t_{n}}^{t} A(s 2) d s 2\right] d s 1\right] d s+\ldots
\end{aligned}
$$

The hypothesis of a linear evolution of $\mathbf{F}$ (see paragraph 4.2) leads to a linear temporal evolution of $[A(t)]$ and as a result $\Omega(t)$ can be analytically determined (at the third order in our study).

Finally the integral [33] is numerically evaluated with a three Gauss points scheme and at this step $\overline{\mathbf{B}}_{\mathbf{e}}\left(t_{n+1}\right)$ is known, and so $\boldsymbol{\sigma}_{\boldsymbol{v}}$ and $\boldsymbol{\pi}_{\boldsymbol{v}}$ using [18] and [13]. 


\subsection{Viscous tangent moduli}

To entirely solve the F.E. problem, $\mathbb{T}_{\mathbf{v}}$ defined in [17], must be evaluated. For this, the neohookean form chosen for $\rho_{0} \psi_{v}$ allows to write ${ }^{6}$ :

$$
\mathbb{T}_{\mathbf{v}}=2 G\left[\frac{\partial \overline{\mathbf{B}}_{\mathbf{e}}{ }^{D}}{\partial \mathbf{F}}\left(2 \mathbf{F}^{-T}+\overline{\mathbf{B}}_{\mathbf{e}}{ }^{D} \cdot \frac{\partial \mathbf{F}^{-T}}{\partial \mathbf{F}}\right]\right.
$$

The second term of the previous expression is easy to evaluate with

$$
\frac{\partial \mathbf{F}^{-T}}{\partial \mathbf{F}}=-\mathbf{F}^{-T} \otimes \mathbf{F}^{-T}
$$

For the first term $\frac{\partial \overline{\mathbf{B}}_{\mathbf{e}}{ }^{D}}{\partial \mathbf{F}}$, having in mind that $\overline{\mathbf{B}}_{\mathbf{e}}{ }^{D}=b(t) \mathbf{N}^{\star}$, it comes that:

$$
\frac{\partial \overline{\mathbf{B}}_{\mathbf{e}}{ }^{D}}{\partial \mathbf{F}}=\mathbf{N}^{\star} \otimes \frac{\partial \delta b(t)}{\partial \mathbf{F}}+b(t) \frac{\partial \mathbf{N}^{\star}}{\partial \mathbf{F}}
$$

The partial derivative of the system [31] with respect of $\mathbf{F}_{i j}$ leads to:

$$
\left\{\begin{array}{c}
\frac{\partial \dot{\delta} s}{\partial \mathbf{F}_{i j}} \\
\frac{\partial \dot{\delta} b}{\partial \mathbf{F}_{i j}}
\end{array}\right\}=[A(t)]\left\{\begin{array}{l}
\frac{\partial \delta s}{\partial \mathbf{F}_{i j}} \\
\frac{\partial \delta b}{\partial \mathbf{F}_{i j}}
\end{array}\right\}+\frac{\partial\{C(t)\}}{\partial \mathbf{F}_{i j}}+\frac{\partial[A(t)]}{\partial \mathbf{F}_{i j}}\left\{\begin{array}{c}
\delta s \\
\delta b
\end{array}\right\}
$$

We can see, that the terms $\frac{\partial\{C(t)\}}{\partial \mathbf{F}_{i j}}, \frac{\partial[A(t)]}{\partial \mathbf{F}_{i j}}$ in [38] and also $\frac{\partial \mathbf{N}^{\star}}{\partial \mathbf{F}}$ in [37] need to be calculated to achieve the determination of [35]. This can be done allowing for:

$$
\begin{aligned}
\frac{\partial \overline{\mathbf{B}}_{\mathbf{e}}^{\star}}{\partial \mathbf{F}}= & \frac{\partial \overline{\mathbf{F}}}{\partial \mathbf{F}}(2)\left(\overline{\mathbf{F}}_{\mid n}^{-1} \cdot \overline{\mathbf{B}}_{\left.\mathbf{e}\right|_{n}} \cdot \overline{\mathbf{F}}_{\left.\right|_{n}}{ }^{-T} \cdot \overline{\mathbf{F}}_{\mid n+1}^{T}\right) \\
& +\left(\overline{\mathbf{F}}_{\left.\right|_{n+1}} \cdot \overline{\mathbf{F}}_{\left.\right|_{n}}{ }^{-1} \cdot \overline{\mathbf{B}}_{\left.\mathbf{e}\right|_{n}} \cdot \overline{\mathbf{F}}_{\mid n}{ }^{-T}\right) \cdot \frac{\partial \overline{\mathbf{F}}^{T}}{\partial \mathbf{F}}
\end{aligned}
$$

The derivative terms of $\overline{\mathbf{F}}$ are defined by

$$
\begin{aligned}
& \frac{\partial \overline{\mathbf{F}}}{\partial \mathbf{F}}=J^{-1 / 3}\left(\mathbb{I}-\frac{1}{3} \mathbf{F} \otimes \mathbf{F}^{-T}\right) \\
& \frac{\partial \overline{\mathbf{F}}^{T}}{\partial \mathbf{F}}=J^{-1 / 3}\left(\mathbf{1} \otimes \mathbf{1}-\frac{1}{3} \mathbf{F}^{T} \otimes \mathbf{F}^{-T}\right)
\end{aligned}
$$

where $\mathbb{I}$ is the fourth order identity tensor.

The resolution of [38] is achieved with a simple forward Euler scheme in order to obtain a fast and simple numerical procedure to evaluate the tangent operator. This could have been realized using the same exponential integration proposed in the resolution of [31] but with an increase of the computing cost.

6. All the tensorial products are defined in appendix. 


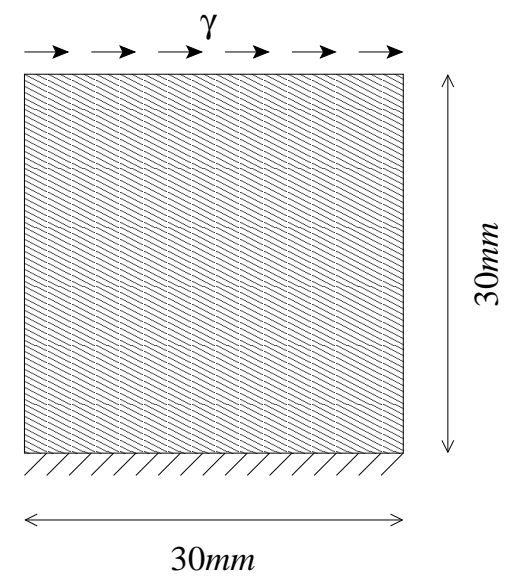

Figure 3. Shear test

\section{Application}

The given example of this section is a classical shear test in plane strain. The considered 2D domain is a square with sides of $30 \mathrm{~mm}$, one side is blocked and the opposite one is subjected to a shear displacement $\gamma=30 \sin (6.6 \pi t)$. It is discretized using ten finite elements per side (quadratic in displacement and linear in pressure). The material parameters are:

$C_{10}=0.97 \mathrm{MPa} \quad C_{01}=-0.42 \mathrm{MPa} \quad G=0.77 \mathrm{MPa} \quad \eta=0.10 \mathrm{MPa} . \mathrm{s} \quad k=1500 \mathrm{MPa}$

The Figure 4(a) shows the influence of the time sub-steps on the solution illustrating the convergence of the proposed algorithm. The differences between the 320 steps and the 17 steps time discretizations seems to be reasonable and are known in the same order in all iterative processes.

Concerning the validity of the results, the shear stress obtained by a direct solving of the Equation [29] or by a first order Taylor expansion of it (i.e. the adopted method) are plotted on the Figure 4(b). The results are nearly the same. But in the Table 1, we can see that for a same number of time steps discretization, we have a CPU time twice longer for the Runge-Kutta-Feldberg method.

Table 1. Comparison of the numerical costs

\begin{tabular}{llll}
\hline method & CPU time (s) & number of sub-steps & time per sub-step (s) \\
\hline radial return relaxation & 85.9 & 320 & 0.268 \\
Runge Kutta Feldberg & 170.8 & 320 & 0.533 \\
\hline
\end{tabular}




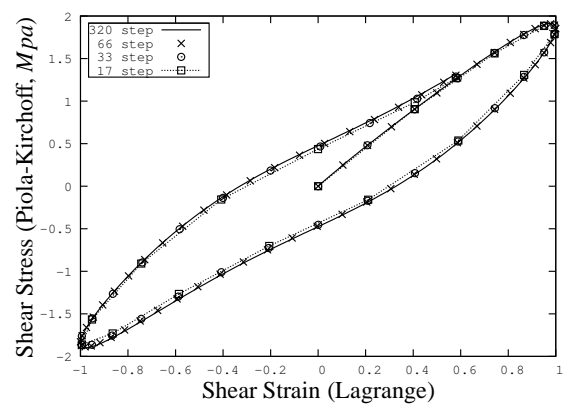

(a) Hysteresis.

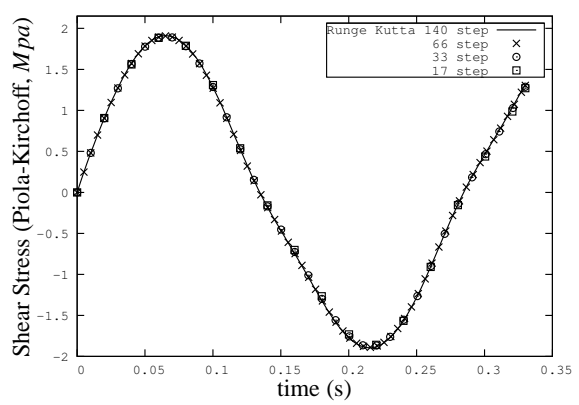

(b) Shear stress versus time.

Figure 4. Results of the numerical shear test

\section{Conclusion}

It has been proposed, in this paper, a new integration scheme based both on the decomposition of the tensorial internal variable in flow directions and flow magnitudes and on a linearization of the differential system obtained. This process is quite general and can be applied on different evolution laws. For a Zener model, this strategy has shown interesting results: the computing time reduction is important compared to a standard iterative integrator, as a Runge-Kutta-Fehlberg scheme. However, further investigations are required to compare this integration scheme to standard ones in more complicated cases. For instance, in the case of a viscoelastic Poynting-Thomson model, the evolution laws are more non-linear and the proposed numerical scheme could failed for large time steps due to the linearization process. Furthermore, the questions of the rate of convergence and the numerical stability have not been investigated in this paper. This will be done in a future communication.

\section{References}

Areias P., Matouš K., " Finite element formulation for modeling nonlinear viscoelastic elastomers", Computer Methods in Applied Mechanics and Engineering, vol. 197, p. 47024717, 2008.

Fancello E., Vassoler J., Stainier L., “ A variational constitutive update algorithm for a set of isotropic hyperelastic-viscoplastic material models", Computer Methods in Applied Mechanics and Engineering, vol. 197, p. 4132-4148, 2008.

Fehlberg E., " Low-order classical runge-kutta formulas with step size control and their application to some heat transfer problems.", Tech. rep.,NASA TR, vol. R-, p. 315, 1969.

Foerch R., Besson J., Cailletaud G., Pivlin P., “ Polymorphic constitutive equations in finite element codes”, Comput. Methods Appl. Mech. Engrg., vol. 141, p. 355-372, 1996.

Iserles. A., Marthinsen A., Nørsett S., " On the implementation of the method of magnus series for linear differential equations.”, BIT Numerical Mathematics, vol. 39, p. 281-304, 1999. 
Lejeunes S., Modélisation de structures lamifiées élastomère-métal à l'aide d'une méthode de réduction de modèles, $\mathrm{PhD}$ thesis, Université d'Aix-Mareille II, 2006.

Nedjar B., " Frameworks for finite strain viscoelastic-plasticity based on multiplicative decomposition. II: Computational aspects", Computer Methods in Applied Mechanics and Engineering, vol. 191, p. 1563-1593, 2002a.

Nedjar B., "Frameworks for finite strain viscoelastic-plasticity based on multiplicative decomposition. Part I: Continuum formulations", Computer Methods in Applied Mechanics and Engineering, vol. 191, p. 1541-1562, 2002b.

Reese S., Govindjee S., “ A theory of finite viscoelasticity and numerical aspects.”, International Journal of Solid and Structures, vol. 35, p. 3455-3482, 1998.

Sidoroff F., " The geometrical concept of intermediate configuration and elastic finite strain", Arch. Mech., vol. 25, n 2, p. 299-309, 1973.

Sidoroff F., "Variables internes en viscoélasticité, 2. Milieux avec configuration intermédiaire", J. Méc., vol. 14, n 4, p. 571-595, 1975.

Sidoroff F., "Variables internes en viscoélasticité, 3. Milieux avec plusieurs configurations intermédiaires”, J. Méc., vol. 15, n 1, p. 85-118, 1976.

Simo J.-C., “ A framework for Finite Strain Elastoplasticity Based on Maximum Plastic Dissipation and the Multiplicative Decomposition: Part II. Computational Aspects.”, Computer Methods in Applied Mechanics and Engineering, vol. 79, p. 1-31, 1988.

Simo J., Hugues T., Computational inelasticity, 2000.

Weber G., Arnand L., " Finite deformation constitutive equations and a time integration procedure for isotropic hyperelastic-viscoplastic solids.", Computer Methods in Applied Mechanics and Engineering, vol. 79, p. 173-202, 1990.

\section{Appendix}

Here are defined the tensorial operators used in this paper:

\begin{tabular}{l|l}
$\cdot$ & the first order contracted tensorial product \\
\hline$:$ & the second order contracted tensorial product \\
\hline$\otimes$ & the classical tensorial product \\
\hline$\odot$ & $\begin{array}{l}\text { a second order tensorial operator } \\
\left([\mathbf{A} \ominus \mathbf{B}]_{i j k l}=A_{i l} B_{k j}\right)\end{array}$ \\
\hline$(2)$ & $\begin{array}{l}\text { a tensorial product between a fourth order tensor } \\
\text { and a second order one }\left([\mathbf{A}(2) \mathbf{B}]_{i j k l}=A_{i o k l} B_{o j}\right)\end{array}$ \\
\hline\|\| & $\begin{array}{l}\text { norm of a second order tensor } \\
\|\mathbf{A}\|=\sqrt{\mathbf{A}: \mathbf{A}}\end{array}$
\end{tabular}

